\title{
Simulação computacional para o problema de dispersão de poluição na lagoa Rodrigo de Freitas
}

\author{
João Paulo Alves Barros ${ }^{1}$ \\ UERJ, Rio de Janeiro, RJ \\ Cristiane Oliveira de Faria ${ }^{2}$ \\ IME/UERJ, Rio de Janeiro, RJ \\ João Frederico da Costa Azevedo Meyer ${ }^{3}$ \\ IMECC/UNICAMP, Campinas, SP
}

\begin{abstract}
Resumo. Como sabemos, um dos maiores problemas do século 21 é a poluição. Neste trabalho apresentamos uma investigação sobre o processo de dispersão da poluição na Lagoa Rodrigo de Freitas e suas conseqüências. Para analisar o processo de poluição, uma proposta de modelagem numérica através da equação difusão-advecção-reação foi considerada. Simulações numéricas são mostradas considerando o Método dos Elementos Finitos para as discretizações espaciais (usando elementos triangulares lineares) combinadas ao método de Crank-Nicolson para que a discretização de tempo seja mostrada. As simulações computacionais foram realizadas monitorando o processo de dispersão de poluição ao longo de 3 dias em 3 cenários diferentes, e além disso, foi realizada uma análise da concentração de poluição em nós específicos ao longo do tempo.
\end{abstract}

Palavras-chave. Problema de transporte, Concentração de poluentes, Método de Elementos Finitos, Método Crank-Nicolson.

\section{Introdução}

A poluição ambiental é um dos maiores problemas deste século e um dos principais fatores que influenciam é o rápido crescimento industrial em escala global aliado à emissão de poluentes. A influência da poluição ocorre de diversas formas como: efeito estufa, alteração em taxas de mortalidades e natalidades de algumas espécies, destruição da camada de ozônio, chuvas ácidas, toxicidade. Todos os fatores citados prejudicam não só a vida do planeta como também a longevidade humana.

Neste trabalho, apresentamos simulações computacionais para o processo de dispersão da poluição da Lagoa Rodrigo de Freitas por efluentes utilizando método de Galerkin combinado com o método de Crank-Nicolson para discretização espacial e temporal, respectivamente. A área da lagoa Rodrigo de Freitas é aproximadamente de $2,2 \mathrm{~km}^{2}$ e se encontra na zona sul da cidade do Rio de Janeiro. Ela é carinhosamente apelidada de "Coração do Rio de Janeiro" devido ao seu formato parecer um coração (veja a Figura 1).

A lagoa é considerada como um dos principais pontos turísticos da cidade do Rio de Janeiro. Em seu entorno encontram-se parques e áreas de lazer para a população. No entanto, é de notório saber que a lagoa se encontra poluída e num estágio em que se banhar nela chega a ser um grave perigo contra a saúde. Desde 2006, a Companhia Estadual de Águas e Esgotos do Rio de Janeiro

\footnotetext{
${ }^{1}$ jpab_7@hotmail.com

2 cofaria@ime.uerj.br

3 joni@ime.unicamp.br
} 
(CEDAE) vem realizando estratégias para conter a poluição da lagoa. Houve inúmeras intervenções com o objetivo de proporcionar melhorias nos sistemas de esgotos sanitários de todas as sub-bacias contribuintes para a Lagoa e com isso iniciar uma recuperação ambiental, já verificada pelos órgãos de fiscalização ambiental do estado.

No entanto, o problema da poluição em lagoas não é um caso particular da Lagoa Rodrigo de Freitas. Em Wolmuth [7] estudos foram feitos para o caso de poluição da represa do Rio Manso, em Prestes [6] para o caso da lagoa do Taquaral, Campinas-SP. Baseado nestes trabalhos e na metodologia proposta em [5], apresentamos simulações computacionais utilizando a mesma metodologia desenvolvida no caso da represa do Rio Manso.

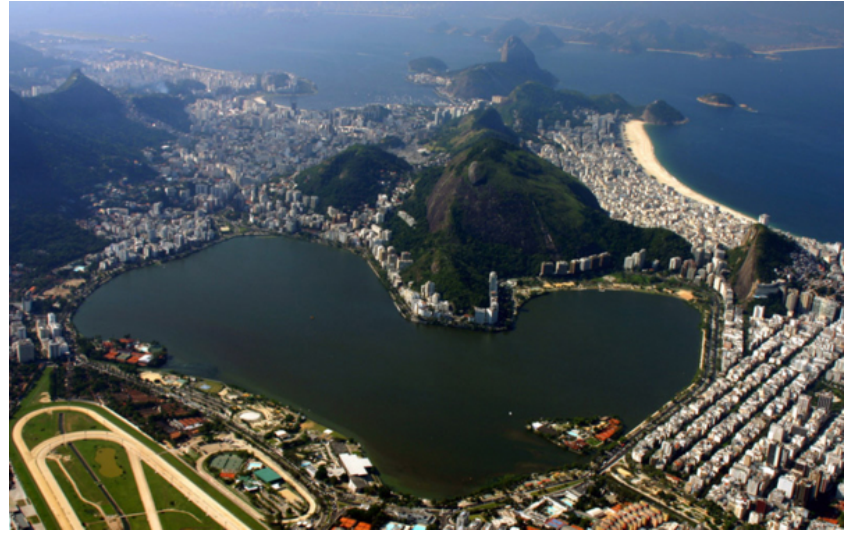

Figura 1: Lagoa Rodrigo De Freitas. Fonte: Jornal O Globo (2019)

Este trabalho está organizado da seguinte forma: na Seção 2 será apresentado o modelo matemático de dispersão de poluição para este tipo de problema com as hipóteses que serão consideradas. Na Seção 3 serão propostos os métodos numéricos para este estudo. Será utilizado na discretização para a variável espacial o método de Galerkin com elementos triangulares lineares e para a variável temporal o método de Crank-Nicolson. Também serão apresentados os testes realizados e resultados computacionais para o problema da lagoa considerando um domínio retangular e da Lagoa Rodrigo de Freitas. Por fim, serão apresentadas as conclusões.

\section{Problema Modelo}

O problema de tratamento e dispersão da poluição será modelado pela equação do transporte com difusão-advecção-reação. O objetivo é encontrar a concentração do poluente $c(t, x, y)$,onde $(x, y) \in \mathbb{R}^{2}$ e $t \in I=[0, T]:$

$$
\left\{\begin{aligned}
\frac{\partial c}{\partial t}-\epsilon \Delta c+\boldsymbol{\beta} \cdot \nabla c+\delta c=f \text { em } & \Omega \\
-\epsilon \frac{\partial c}{\partial \eta}=0 \text { em } & \Gamma .
\end{aligned}\right.
$$

O parâmetro $\epsilon$ é a constante de difusibilidade, $\boldsymbol{\beta}=(u, w)$ é a velocidade da superfície da água, $\delta$ é definida como a taxa de decaimento no meio aquático e $f$ é o termo fonte, que pode ser uma ou várias fontes de poluição ou sumidouros, pontuais ou não.

A condição de fronteira está atrelada ao fato da lagoa perder poluentes em suas bordas $\Gamma$. Neste estudo consideraremos apenas o caso de um agregado, o concreto, que foi utilizado para revestir a borda de toda a lagoa levando que a perda de poluente na borda é igual a zero. 


\section{Discretização do Problema}

O mapeamento geométrico da região da Lagoa Rodrigo de Freitas foi feita utilizando o software Google Earth ${ }^{\circledR}$. Foram obtidas as coordenadas necessárias para a fronteira aproximada da original através de segmentos de retas. Com o auxílio do software livre $G M S H^{4}$, foi gerada uma malha considerando elementos triangulares (veja Figura 2).

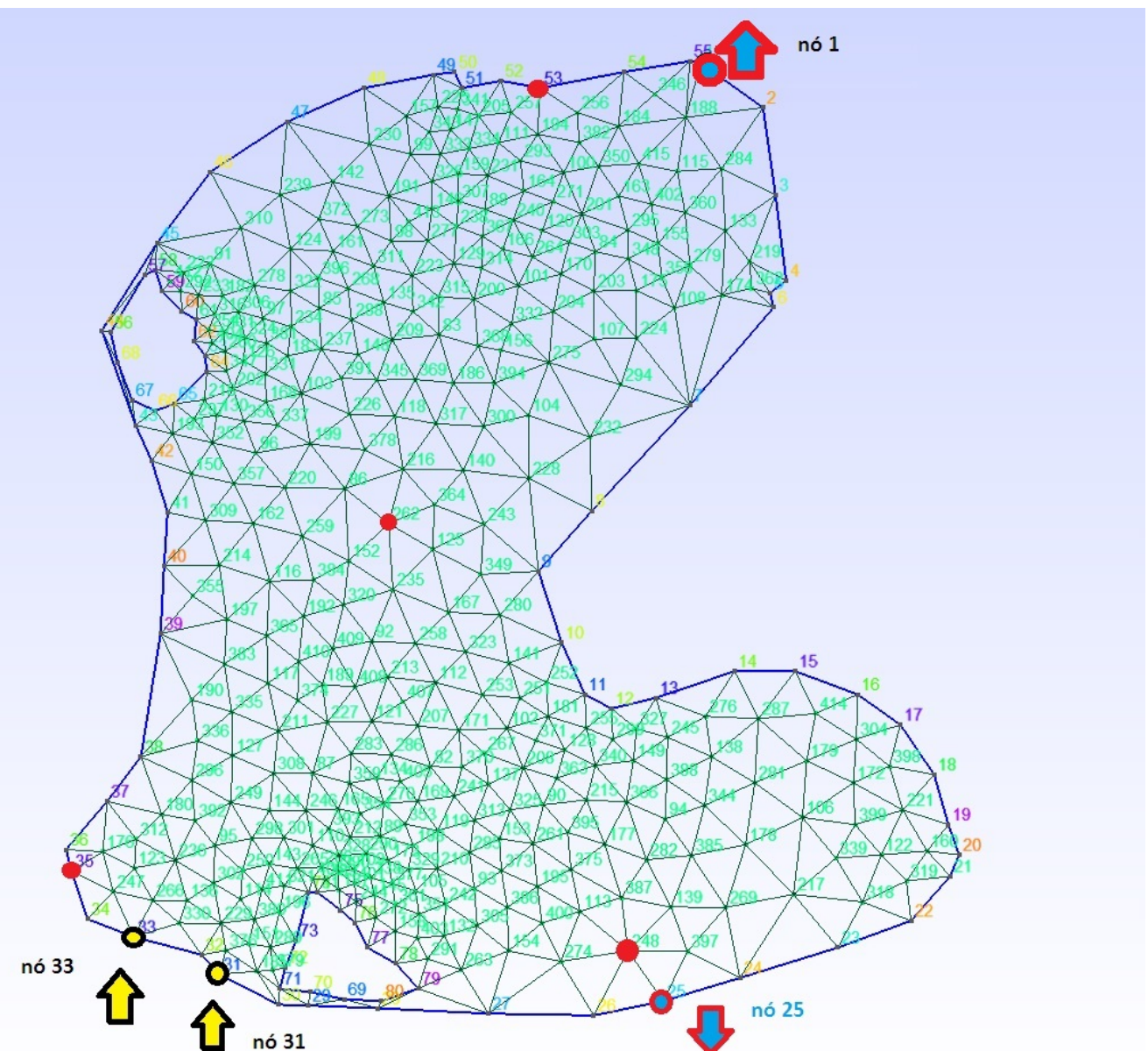

Figura 2: Malha gerada utilizando o GMSH.

A malha gerada pelo GMSH aproxima a Lagoa Rodrigo de Freitas com 519 elementos triangulares de ordem linear (P1) e 415 nós. Na Figura 2 também seguem destacados em vermelho os nós que serão utilizados para análise de concentração de poluição, as flechas azuis são os nós onde ocorre saída de poluente e as flechas em amarelo são os nós que são fonte de poluição. Todos serão explicitados para cada estudo de caso na Tabela 4.

Dado $H_{h}$, um subespaço vetorial de $H^{1}(\Omega)$, de dimensão finita $\mathrm{N}$, define-se a base $K=\left\{\phi_{1}(x, y)\right.$, $\left.\phi_{2}(x, y), \ldots, \phi_{N}(x, y)\right\}$.

Sendo assim, queremos encontrar $c_{h} \in H_{h}$ tal que:

$$
\left(\frac{\partial c_{h}}{\partial t}, v_{h}\right)+\epsilon\left(\nabla c_{h}, \nabla v_{h}\right)+\left(\boldsymbol{\beta} \cdot \nabla c_{h}, v_{h}\right)+\left(\delta c_{h}, v_{h}\right)=\left(f, v_{h}\right) \quad, \forall v_{h} \in V_{h}
$$

\footnotetext{
${ }^{4}$ Disponível em: <http://gmsh.info/>. Acesso em 3 dez. 2019.
} 
Aplicando o Método de Crank-Nicolson como em (ver [7]), obtemos o seguinte sistema linear o passo de tempo $n+1$ :

$$
A c^{n+1}=B c^{n}+d
$$

onde:

- $A=\left[a_{i j}\right], B=\left[b_{i j}\right], d=\left[d_{i}\right]$,

- $a_{i j}=\left[\left(1+\frac{\delta \Delta t}{2}\right)\left(\phi_{j}, \phi_{i}\right)+\frac{\epsilon \Delta t}{2}\left(\nabla \phi_{j}, \nabla \phi_{i}\right)+\frac{u \Delta t}{2}\left(\frac{\partial \phi_{j}}{\partial x}, \phi_{i}\right)+\frac{w \Delta t}{2}\left(\frac{\partial \phi_{j}}{\partial y}, \phi_{i}\right)\right]$

- $b_{i j}=\left[\left(1-\frac{\delta \Delta t}{2}\right)\left(\phi_{j}, \phi_{i}\right)-\frac{\epsilon \Delta t}{2}\left(\nabla \phi_{j}, \nabla \phi_{i}\right)-\frac{u \Delta t}{2}\left(\frac{\partial \phi_{j}}{\partial x}, \phi_{i}\right)-\frac{w \Delta t}{2}\left(\frac{\partial \phi_{j}}{\partial y}, \phi_{i}\right)\right]$,

- $d_{i}=\Delta t\left(f, \phi_{i}\right)$.

em que $\Delta x$ e $\Delta y$ são os espaçamentos da malha na horizontal e na vertical, respectivamente e $\Delta t$ o espaçamento correspondente à malha temporal. O sistema acima será resolvido iterativamente no tempo, a partir da condição inicial $c_{0}$, previamente fornecida.

\section{Simulação computacional}

Nesta seção apresentamos simulações feitas para alguns casos propostos de dispersão de poluição na lagoa. Na Tabela 2, coletamos os valores dos parâmetros e constantes utilizados nos testes. É importante ressaltar que os valores da constante de difusibilidade, da velocidade do vento, do decaimento e da condição de fronteira utilizados ao longo deste estudo foram retirados de Wolmuth [7] e Prestes [6]. Na Tabela 1, para cada caso é mostrado a numeração dos nós utilizados como saída de poluentes ou fonte de poluição e cada caso monitora o processo de poluição ao longo de 3 dias da lagoa. A localização destes pode ser vista na Figura 2.

Tabela 1: Localização dos nós utilizados como saída de poluentes ou fonte de poluição.

\begin{tabular}{|c|c|c|c|}
\hline & Caso 1 & Caso 2 & Caso 3 \\
\hline Fontes de Poluição & 31 & 31 e 33 & 31 e 33 \\
\hline Sumidouros & 25 & 25 & 1 e 25 \\
\hline
\end{tabular}

Tabela 2: Valores dos parâmetros utilizados nos testes.

\begin{tabular}{|c|c|c|c|}
\hline$\epsilon$ & $0.4 \mathrm{~km}^{2} / \mathrm{h}$ & Velocidade & $1.9 \mathrm{~km} / \mathrm{h}$ \\
\hline$w$ & $0.00360 \mathrm{~km} / \mathrm{h}$ & Número de Dias & 3 \\
\hline$u$ & $0.00360 \mathrm{~km} / \mathrm{h}$ & $\mathrm{N}_{e}$ & 751 \\
\hline$\delta$ & $10^{-10} \mathrm{~h}^{-1}$ & nós & 415 \\
\hline $\mathrm{C}_{0}$ & 0.14 & Tempo Final & $72 \mathrm{~h}$ \\
\hline$\Delta t$ & $0.024 \mathrm{~h}$ & Passos de tempo & 1000 \\
\hline Fontes de Poluição & $0.14 \mathrm{~kg} / \mathrm{h}$ & Sumidouros & $-0.10 \mathrm{~kg} / \mathrm{h}$ \\
\hline
\end{tabular}

Na Figura 3, apresentamos as simulações dos três cenários descritos para a dispersão da concentração de poluentes na lagoa ao longo de 3 dias. Comparando o segundo caso, que teve um cenário com duas fontes de poluição e um sumidouro, com o primeiro caso que só possuia uma fonte e um sumidouro, podemos notar que a a concentração de poluentes na lagoa foi maior que no primeiro caso. A adição de mais uma fonte de poluição teve o efeito esperado. No terceiro caso, foi adicionado mais um sumidouro, a adição deste fez com que houvesse uma filtragem maior de 


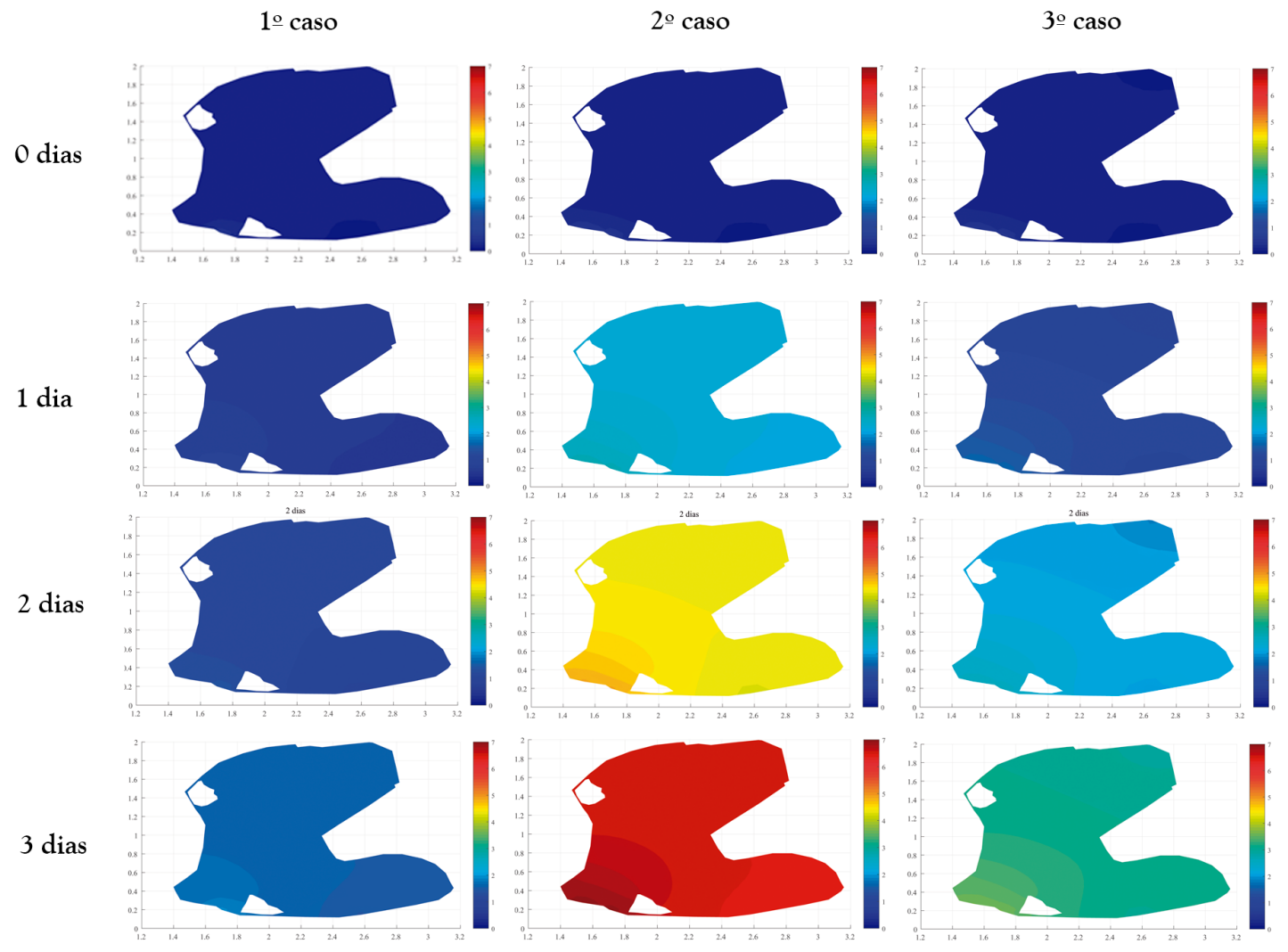

Figura 3: Casos de poluição na Lagoa Rodrigo de Freitas ao longo de 3 dias: (a) $1^{\circ}$ caso, (b) $2^{\circ}$ caso e (c) $3^{\circ}$ caso.

poluente fazendo com que diminuisse a concentração total de poluentes da lagoa. Pode-se dizer que a concentração total caiu em aproximadamente $50 \%$ comparado ao segundo cenário.

Com o intuito de explorar melhor o problema e diferentemente das primeiras simulações que visavam apenas analisar a lagoa e sua concentração total, nesta segunda abordagem será analisada a concentração, dos nós 1, 262, 25 e 248 ao longo de 3 dias em cada caso. Na Figura 4 pode-se observar a concentração dos nós utilizados nos testes realizados na lagoa. Pode-se observar que no segundo caso com duas fontes de poluição, uma a mais que o primeiro, e mantendo o mesmo sumidouro, ocorreu um aumento de concentração em cada nó, o que era esperado observando o teste anterior realizado. No terceiro caso, com a adição de um sumidouro, é possível observar que a concentração de poluição dos nós foi menor que no segundo caso, o que era esperado ocorrer se for observado a Figura 3. A palheta de cores da Figura 3 mostra que a lagoa teve cerca de $50 \%$ de queda de concentração de poluente e observando a Figura 4 vemos que os nós neste caso obtiveram praticamente a mesma queda na concentração.

Estudos analisando a Matriz Global do problema, análises de casos em que a condição de Péclet não é obedecida além de testes variando a quantidade de elementos podem ser vistos em [1]. 
1으 caso
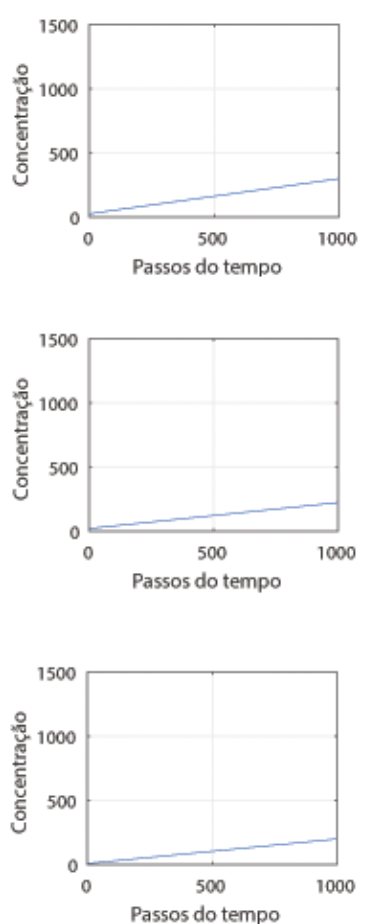

Nó 248

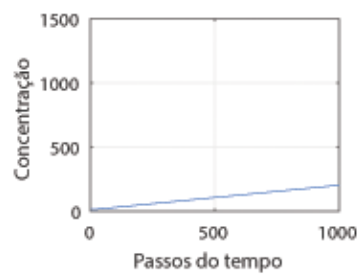

20 caso
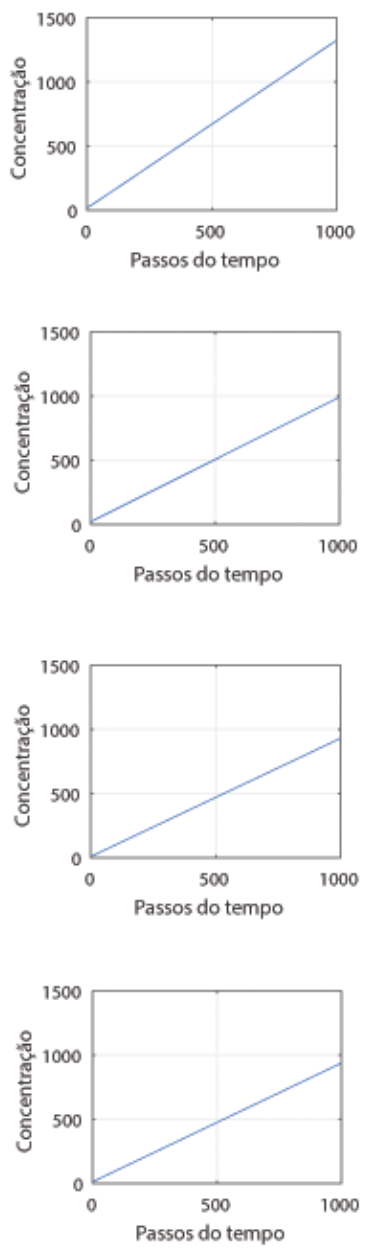

30 caso
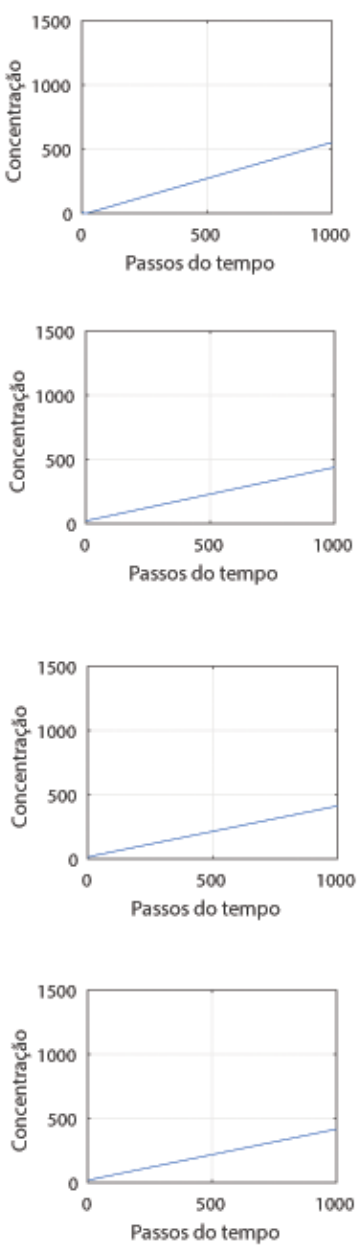

Figura 4: Concentração ao longo do tempo nos nós da Figura (a) $1^{\circ}$ caso, (b) $2^{\circ}$ caso e (c) $3^{\circ}$ caso. 2.

\section{Conclusões}

É possível concluir que, à medida que se aumenta o número de fontes de poluições, a concentração de poluentes na lagoa aumenta. O inverso ocorre com a adição de sumidouros, aumentandose o número de sumidouros, aumenta a filtragem de poluente e consequentemente diminui a concentração total de poluentes.

A utilização do GMSH, Google Earth ${ }^{\circledR}$ e de uma triangularização irregular nos deu uma melhor aproximação para a região de estudo. Sob este ponto de vista, é possível vislumbrar algumas abordagens para futuros projetos, no sentido de aprimorar o programa ou até mesmo o estudo com a possibilidade de trabalhar no $\mathbb{R}^{3}$ como no caso do trabalho proposto por Diniz [5] ou utilizar Stokes para gerar a circulação da Lagoa.

O algoritmo utilizado é efetivo, assim sendo, pode ser utilizado para simular outras regiões, inserindo suas respectivas malhas, coordenadas dos nós e das fronteiras. 


\section{Agradecimentos}

O presente trabalho foi realizado com apoio da Coordenação de Aperfeiçoamento de Pessoal de Nível Superior - Brasil (CAPES) - Código de Financiamento 001.

\section{Referências}

[1] Barros, J. P. A. Soluções numéricas para o problema de dispersão de poluição na Lagoa Rodrigo de Freitas, Dissertação de Mestrado, UERJ, 2019.

[2] Charny, C. K. Mathematical models of bioheat transfer. Advances in Heat Transfer - Bioengineering Heat Transfer, 1992. DOI: 10.1016/S0065-2717(08)70344-7

[3] Equer, D. B. Modelagem, aproximação e simulações computacionais de impacto ambiental com difusibilidade variável: um estudo de caso, Dissertação de Mestrado, Unicamp, 2017.

[4] Krindges, A. Modelagem e simulação computacional de um problema tridimensional de difusão-advecção com o uso de Navier Stokes, Tese de Doutorado, Unicamp, 2006.

[5] Meyer, J. F. C. A. and Diniz, G. L. Pollutant dispersion in wetland systems: Mathematical modelling and numerical simulation, Ecological Modelling, 2007. DOI: 10.1016/j.ecolmodel.2006.08.005.

[6] Prestes, M. F. B. e Meyer, J. F. C. A. A influência da dispersão de material impactante em meio aquático na dinâmica populacional entre duas espécies de peixes: modelo matemático, aproximação numérica e simulação computacional - Lagoa do Taquaral - Campinas/SP. Biomatemática (Unicamp),24:65-76, 2014.

[7] Wolmuth, D. L. Modelagem e simulações do comportamento evolutivo de poluentes em corpos aquáticos de grande extensão: O caso da represa do Rio Manso, Dissertação de Mestrado, Unicamp, 2009. 